\title{
Bilateral Vocal Cord Paralysis Due to Prolonged Intubation in COVID-19 Patients-Case Reports
}

\author{
Bathula SSR*, Megan K and Andrew R \\ Department of Otolaryngology, Head and Neck Surgery, Detroit Medical Center, USA
}

*Corresponding author: Samba BSR, MD, Department of Otolaryngology, Head and Neck Surgery, Detroit Medical Center, Detroit, MI 48201, USA, Tel: 3139669471; Email: sambasivareddy@yahoo.com

\section{Case Report}

Volume 5 Issue 2

Received Date: September 18, 2020

Published Date: October 05, 2020

DOI: $10.23880 /$ ooaj-16000200

\section{Abstract}

Patients typically undergo tracheostomy tube placement after prolonged intubation in order to decrease the risks of endotracheal intubation including laryngeal stenosis, swelling and vocal cord paralysis. This is done prior to 21 days of intubation. During the current coronavirus pandemic there has been an increase in endotracheal intubations and delay in tracheostomy tube placement beyond the 21 days secondary to new recommendations. Presented here are two cases at our institution of patients with the novel coronavirus disease (COVID-19) and prolonged ( $>21$ days) intubation prior to tracheostomy tube placement that have bilateral vocal cord paralysis without laryngeal or tracheal stenosis after extubation. Although the new recommendations of delayed tracheostomy tube placement are intended to decrease spread, there may be increased incidence of unintended effects to the patients.

Keywords: Vocal Cord; Vocal Cord paralysis; COVID-19; Intubation

Abbreviations: COPD: Chronic Obstructive Pulmonary Disease; CHF: Congestive Failure.

\section{Introduction}

Tracheostomy procedures are usually performed before or at 21 days of intubation as recommended by the American College of Chest Physicians. This is because it was discovered that patient who were intubated for greater than 21 days had an increased risk of laryngeal injuries including laryngeal stenosis, swelling, and vocal cord paralysis [1] with the most common injury being subglottic stenosis [2].

With the outbreak of the novel coronavirus disease 2019 (COVID-19) there has been an increase in endotracheal intubations secondary to respiratory decompensation. COVID-19, a highly contagious RNA beta virus, also known as severe acute respiratory syndrome coronavirus 2 (SARSCoV-2), can cause pneumonia and acute respiratory distress syndrome in patients causing them to require critical care support including endotracheal intubation and being in the prone position for greater than 3 weeks [3]. Due to the highly virulent nature of COVID-19 and its spread through respiratory particles, there is a high chance of spread to healthcare workers when turning the patient or if accidental decannulation occurs. This has resulted in postponement of the tracheostomy tube placement beyond the recommended 21-day period [4,5]. Although, postponement of tracheostomy tube placement in these patients may help reduce the spread, it may also have unintended effects on the patient. Here, we report two cases of bilateral vocal cord paralysis after prolonged intubation secondary to COVID-19 pneumonia.

\section{Case Presentation}

Charts of two patients were comprehensively analyzed by the team of otolaryngology resident, student and the primary author who served as the attending physician 
(Samba Siva R Bathula, SSB).

\section{Case One}

76 year old, COVID-19 PCR positive female, with a prior history of congestive failure (CHF) and chronic obstructive pulmonary disease (COPD) was intubated with a standard 7.0 endotracheal tube for acute respiratory failure. Patient then underwent percutaneous tracheostomy tube placement after 23 days of intubation. Five months after tracheostomy tube placement and removal of the endotracheal tube the patient was found to have complete bilateral vocal cord paralysis. The patient had no evidence of subglottic stenosis.

\section{Case Two}

53 year old, COVID-19 PCR positive female, with a prior history of low-grade B-cell non-Hodgkin's lymphoma treated four years ago, was intubated with a standard 7.0 endotracheal tube for acute respiratory failure. She was extubated after standard tracheostomy was performed 45 days after endotracheal intubation. Four months after tracheostomy tube placement and removal of endotracheal tube the patient was found to have complete bilateral vocal cord paralysis. Again, this patient had no evidence of subglottic stenosis similar in presentation to the previous patient (Figure 1).

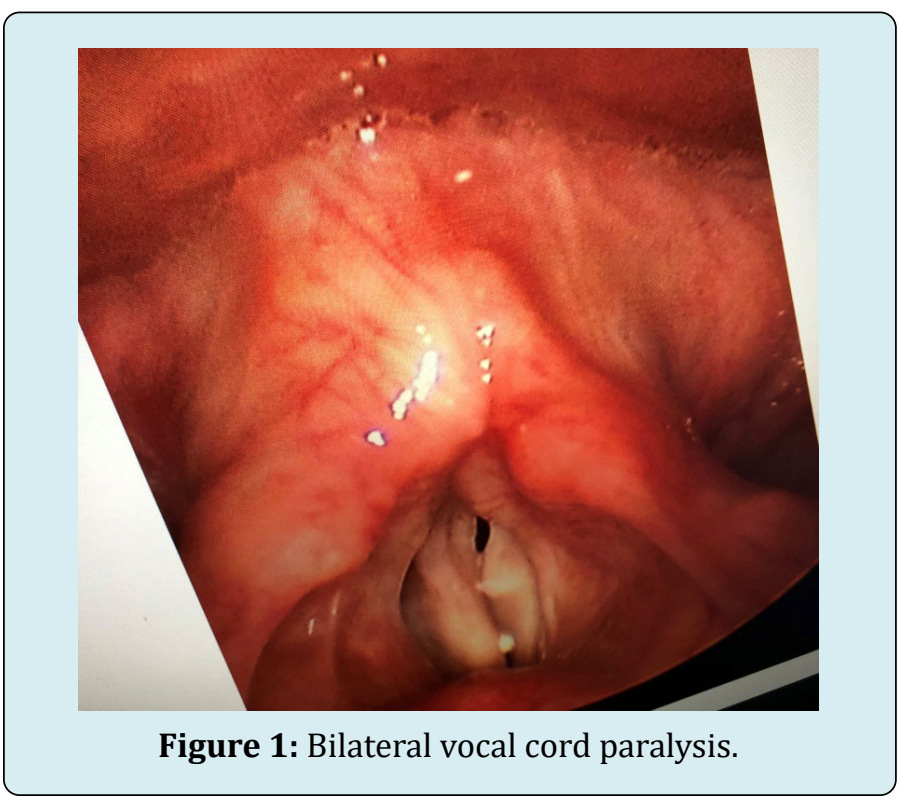

\section{Discussion}

The recurrent laryngeal nerve, a branch of the vagus nerve, supplies motor innervation to the vocal cords. Vocal cord paralysis is commonly seen whenever there is an injury to the recurrent laryngeal nerve due to its torturous course.
The most common injury to the nerve results in unilateral vocal cord paralysis, making bilateral paralysis a rare complication [6].

Bilateral vocal cord paralysis in adult patients is most commonly seen in post-thyroidectomy surgery patients and can be rarely seen in bronchogenic carcinoma patients $[7,8]$. Both conditions require a major head and neck surgery resulting in higher risk to the recurrent laryngeal nerve compared to routine endotracheal intubation. The risk of injury secondary to endotracheal intubation comes from the duration of intubation. According to Kikura, et al. increased endotracheal intubation duration causes a higher chance of vocal cord injury leading to vocal cord paralysis. However, these patients typically regain vocal cord function within two to three months [2].

Case one and two both show an increased duration of endotracheal intubation beyond the recommended guidelines at 23 and 45 days respectively. This was due to the patient's positive COVID-19 status and the new recommendation to delay tracheostomy tube placement due to the high virulence of the disease causing increased risk of spread to healthcare workers [4]. As a result both patients developed bilateral vocal cord paralysis which did not resolve after four months of tracheostomy tube placement. Surprisingly, both patients did not have subglottic stenosis which has been cited as the most common tracheal complication of prolonged intubation [1].

With the increasing cases of COVID-19 it is suspected that there will be an increase in bilateral vocal cord paralysis secondary to prolonged endotracheal intubation. As stated previously, bilateral vocal cord paralysis is usually only seen in extensive head and neck cases including bronchogenic carcinoma or thyroid removal where the nerve is at a greater risk. Increased occurrences of bilateral vocal cord paralysis secondary to COVID-19 prolonged intubations pose the idea that better guidelines or management to minimize patient airway trauma with reduced viral spread to healthcare workers need to be put in place.

\section{Conclusion}

Bilateral vocal cord paralysis is rarely seen from prolonged intubation however the risk increases with duration of intubation. Due to new tracheostomy guidelines, delayed tracheostomy tube placement is occurring in order to reduce the risk of spread to healthcare workers. We presented two patients with prolonged intubation secondary to COVID-19 and delayed tracheostomy tube placement that have bilateral vocal cord paralysis. Further recommendations are needed to address a possible increase in vocal cord injury secondary to prolonged intubation in COVID-19 patients. 


\section{References}

1. Cheung NH, Napolitano LM (2014) Tracheostomy: Epidemiology, Indications, Timing, Technique, and Outcomes. Respir Care 59(6): 895-919.

2. Kikura M, Suzuki Y, Itagaki T, Sato T, Nishino J (2015) Vocal cord paralysis associated with tracheal intubation: incidence, risk analysis, and classification of severity. The Japanese Journal of Anesthesiology 64(1): 57-59.

3. Guan WJ, Ni ZY, Hu Y, Liang WH, Ou C, et al. (2020) Clinical characteristics of Coronavirus disease 2019 in China. N Engl J Med 382: 1708-1720.

4. Sommer DD, Engels PT, Weitzel EK, Khalili S, Corsten $\mathrm{M}$, et al. (2020) Recommendations from the CSO-HNS taskforce on performance of tracheotomy during the COVID-19 pandemic. J Otolaryngol Head Neck Surg 49(1): 23.
5. To KK, Tsang OT, Leung WS, Tam AR, Wu TC, et al. (2020) Temporal profiles of viral load in posterior oropharyngeal saliva samples and serum antibody responses during infection by SARS-CoV-2: an observational cohort study. Lancet Infect Dis 20(5): 565-574.

6. Özbal Koç AE, Türkoğlu SB, Erol O, Erbek S (2016) Vocal cord paralysis: What matters between idiopathic and non-idiopathic cases?. Kulak Burun Bogaz Ihtis Derg 26(4): 228-233.

7. Reiter R, Pickhard A, Smith E, Hansch K, Weber T, et al. (2015) Stimmlippenparesen--Untersuchungen an einem Kollektiv von 400 Patienten [Vocal cord paralysis--analysis of a cohort of 400 patients]. Laryngorhinootologie 94(2): 91-96.

8. Baumann MH, Heffner JE (1989) Bilateral vocal cord paralysis with respiratory failure. A presenting manifestation of bronchogenic carcinoma. Arch Intern Med 149(6): 1453-1454. 